\title{
Fospropofol disodium injection for the sedation of patients undergoing colonoscopy
}

\author{
Benjamin E Levitzky' \\ John J Vargo \\ 'Department of Gastroenterology \\ and Hepatology, ${ }^{2}$ Section of \\ Therapeutic and Hepatobiliary \\ Endoscopy, Department of \\ Gastroenterology and Hepatology, \\ Cleveland Clinic Foundation, \\ Cleveland, Ohio, USA
}

\begin{abstract}
Sedation plays a central role in making colonoscopy tolerable for patients and feasible for the endoscopist to perform. The array of agents used for endoscopic sedation continues to evolve. Fospropofol (FP), a prodrug of propofol with a slower pharmacokinetic profile, is currently under evaluation for use during endoscopic procedures. Preliminary data suggests that FP dosed at $6.5 \mathrm{mg} / \mathrm{kg}$ is well tolerated by most patients with perineal paresthesias being the most commonly experienced adverse effect. This article will examine the current literature on the use of FP for the sedation of patients undergoing colonoscopy, highlighting the pharmacokinetics, pharmacodynamics, risks, and common adverse events associated with the novel sedative/hypnotic.
\end{abstract}

Keywords: fospropofol, Aquavan, propofol, sedation, colonoscopy

\section{Introduction}

Sedation during colonoscopy is important for making the procedure comfortable for patients and feasible for the gastroenterologist. The use of procedural sedation enables the endoscopist to perform a thorough exam while minimizing patient anxiety and risk of injury. All major US gastroenterology societies support the administration of sedation by properly trained gastroenterologists performing routine endoscopic procedures on average risk patients. Since its introduction in the 1980 s, propofol (Diprivan $^{\circledR}$, 2,6-diisopropylphenol; AstraZeneca Pharmaceuticals) has been used for an increasing number of procedures due to its rapid onset and short elimination half-life. Propofol has several advantages over benzodiazepines and opiates including a faster onset of action, high patient satisfaction, and a more rapid recovery to full levels of consciousness with minimal residual sedative effects (Sipe et al 2002). When used as an infusion, propofol produces a rapid peak plasma concentration that may quickly induce deep sedation. Due to this rapid pharmacokinetic profile, concerns have been expressed regarding propofol's potential to induce deeper levels of sedation than intended when patients are improperly monitored. For this reason the product label endorsed by the US Food and Drug Administration (FDA) has limited the use of propofol to anesthesia specialists (AstraZeneca Pharmaceuticals 2004). In this environment, attention has been drawn to formulations of propofol that may be more acceptable for non-anesthesiologist administration.

Fospropofol disodium (Aquavan ${ }^{\circledR}$ injection; MGI Pharma, Inc, Bloomington, MN, USA) is a water-soluble prodrug of propofol currently under investigation for diagnostic procedures. Fospropofol (FP) is rapidly hydrolyzed by endothelial alkaline phosphatases in vivo after intravenous administration, releasing propofol, phosphate, and formaldehyde (Figure 1). FP-derived propofol is the active compound that provides sedation and has a slower pharmacokinetic and pharmacodynamic profile than propofol lipid emulsion. Other metabolites of FP do not accumulate above endogenous levels (Fechner et al 2003). This article will review the current understanding of FP's pharmacokinetics, pharmacodynamics, risks, and benefits for sedation during colonoscopy. It will also examine the recent clinical trials utilizing FP for endoscopic sedation. 


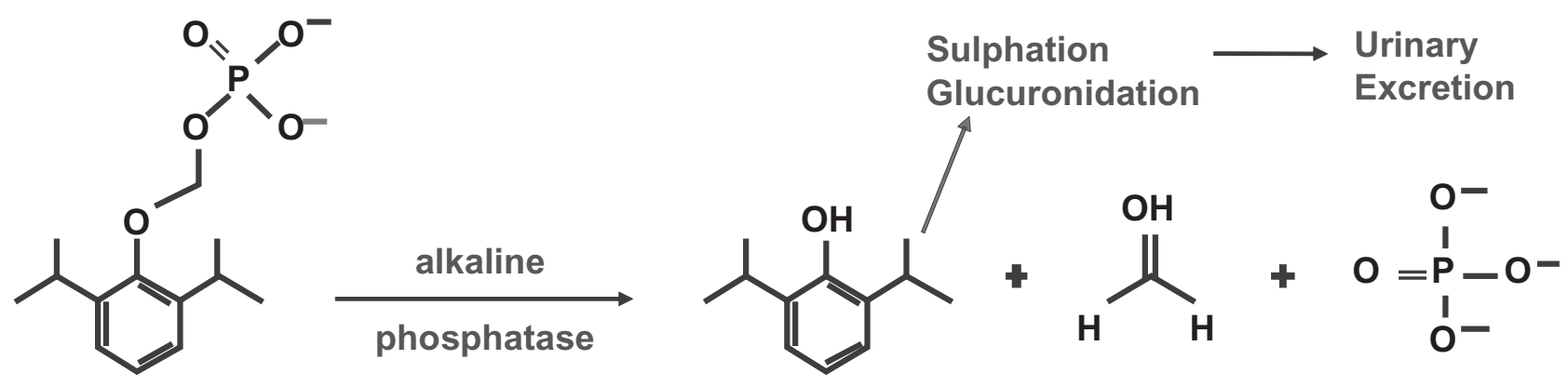

fospropofol disodium

Propofol Formaldehyde Phosphate

Figure I Metabolism of fospropofol.

\section{Potential advantages of fospropofol}

One potential advantage of FP is that the manufacturer is seeking approval from the FDA for FP administration by nonanesthesia specialists (Rex 2006). This may permit a wider spectrum of properly trained physicians to use FP than propofol emulsion. Another potential advantage of FP over propofol relates to its nonlipid formulation. Propofol emulsion, as a lipidbased formula, is prone to contamination and is a poor choice for patients with hyperlipidemia. These risks are ameliorated by FP which does not utilize a lipid suspension for compound delivery. The pharmacokinetic profile of FP has both advantages and disadvantages. The advantage is that its slower profile allows for an ease of administration that requires less frequent bolusing of medication for brief endoscopic procedures (Rex 2006). While this potential advantage may have relevance in a busy, clinical setting, it must be put into the context of a slower onset of action and a prolonged recovery time.

\section{Risks of fospropofol}

The most frequently observed adverse events from FP administration are self-limited, mild to moderate discomfort on infusion, perineal paresthesias, and pruritus (Rex et al 2007a, b; Cohen 2008). In one open label, phase II clinical trial in patients undergoing colonoscopy, transient mild to moderate perineal tingling, itching, or burning were experienced in $85 \%$ of patients (Pruitt et al 2005). While the mechanism behind this reaction remains unknown, other intravenous medications that contain phosphate esters such as fosphenytoin and dexamethasone have been noted to cause similar side effects (Luer 1998; Perron et al 2003). FP may cause small increases in the levels of serum phosphorus, although no clinically significant adverse events from hyperphosphatemia have been reported (Fechner et al 2004; Gibiansky et al 2005).
Although FP is associated with a hemodynamic profile comparable to that of propofol, one study noted that FP produced an early tachycardia in healthy volunteers (Struys et al 2005). A note of caution is necessary as deeper than intended sedation with fospropofol (due to a longer halflife) should be expected to last longer than deep sedation inadvertently induced with propofol. As with all agents used for sedation, excessive dose escalation can lead to deeper than intended degrees of sedation and concomitant apnea or airway compromise. Respiratory depression remains a clinical precursor to more serious compromise in cardiovascular function, and adequate monitoring of patients undergoing sedation remains essential (Rex et al 2005).

\section{Drug combinations}

Propofol emulsion and FP can both be titrated to moderate sedation when co-administered with narcotics, benzodiazepines, or both (Cohen et al 2004; Rex 2006; VanNatta and Rex 2006). As FP is a pure sedative/hypnotic without analgesic properties, it has recently been studied with co-administration of an opiate to improve pain control and to allow for successful targeting of moderate sedation (Rex 2006). Fentanyl $50 \mu \mathrm{g}$ prior to initial bolus dosing of FP is the analgesic dose favored in recent clinical trials (Rex et al 2007a, b; Cohen 2008).

\section{Pharmacokinetics of fospropofol}

After intravenous administration of FP, propofol is released from its prodrug by the enzymatic activity of alkaline phosphatases in the vascular endothelium (Figure 1) (Fechner et al 2003). This mechanism of action provides a controlled liberation of propofol with a smooth increase in plasma propofol concentration. Despite generating the same functional compound as propofol emulsion, FP-derived propofol has a different pharmacodynamic and pharmacokinetic profile 
(Figure 2) (Shah et al 2007). Notably, FP has an extended elimination half-life, a longer residence time, and a delayed onset of action (Schywalsky et al 2003; Fechner et al 2004).

Propofol is eliminated in three phases. The first phase of elimination $\left(\mathrm{t}^{1 / 2} \alpha\right)$ occurs within $2-3$ minutes after intravenous administration and represents the delivery of propofol throughout body tissues. The second phase of elimination $\left(\mathrm{t}^{1} / 2 \beta\right)$ is a longer period lasting 30-60 minutes and represents the metabolism of propofol. The final stage of elimination $\left(\mathrm{t}^{1} / 2 \gamma\right)$ is a much longer stage of variable duration. This stage can last $2-45$ hours and represents ongoing metabolism of drug as it slowly emerges from poorly perfused adipose tissue (Gan 2006).

\section{Pharmacodynamics}

Preliminary studies suggested that at equal plasma propofol concentrations, FP-derived propofol yielded a stronger pharmacodynamic effect than that seen with propofol emulsion without a significant hysteresis (Fechner et al 2004). However, a later study found that the pharmacodynamic effect of propofolemulsion and FP-derived propofol were similar once adjusted for intracerebral propofol concentration (Levitt and Schnider 2005). Continuous intravenous infusion of FP produces dosedependent sedation as measured by the Modified Observer's Assessment of Alertness/Sedation (MOAA/S) score and the bispectral index (BIS) (Fechner et al 2005). Fechner and colleagues demonstrated that FP-delivered propofol at a plasma concentration of $1.8 \mu \mathrm{g} / \mathrm{mL}$ or a bolus of $10 \mathrm{mg} / \mathrm{kg}$ were most likely to achieve a MOAA/S score of 3, equivalent to the moderate degree of sedation targeted for endoscopic procedures (Fechner et al 2005). Other researchers have demonstrated a

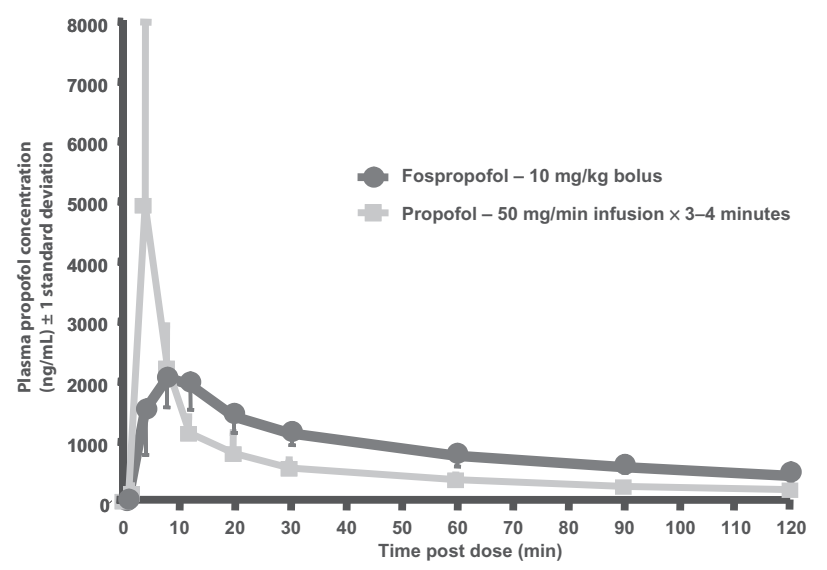

Figure 2 Mean $( \pm \mid \mathrm{SD}$ ) plasma propofol concentration after $10 \mathrm{mg} / \mathrm{kg}$ bolus dose of fospropofol vs $50 \mathrm{mg} / \mathrm{min}$ infusion of propofol $\times 3-4$ minutes in 12 healthy subjects. Reprinted with permission from Shah A, Fechner J, Struys M, et al 2007. Differential PK/PD of propofol after intravenous fospropofol and diprivan in healthy subjects. Anesthesiology, 107:A46. curvilinear relationship between escalating doses of FP and pharmacodynamic effect, with doses up to $20 \mathrm{mg} / \mathrm{kg}$ producing a linear response (Gibiansky et al 2005).

\section{Trials using fospropofol sedation during endoscopic procedures}

To date there are no large scale trials comparing outcomes of FP with those of propofol. However, multiple clinical trials have compared the use of FP with benzodiazepines and opiates for endoscopic sedation. One randomized, double-blind, multicenter trial examined rates of sedation success in 127 adult subjects undergoing colonoscopy using FP $(2,5,6.5$, or $8 \mathrm{mg} / \mathrm{kg})$ with American Society of Anesthesiologist (ASA) risk classes of 1 through 4 (Cohen et al 2006; Cohen 2008). Sedation success was defined as completion of the colonoscopy without a need for mechanical/manual ventilation and without the need for alternative sedative medication. Sedation success rates were dose dependent, with $24.0 \%$ in the $2 \mathrm{mg} / \mathrm{kg}$ arm, $34.6 \%$ in the $5 \mathrm{mg} / \mathrm{kg}$ arm, $69.2 \%$ in the $6.5 \mathrm{mg} / \mathrm{kg}$ arm, and $95.8 \%$ in the $8 \mathrm{mg} / \mathrm{kg}$ arm achieving this endpoint $(\mathrm{p}<0.001)$. No patient required assisted ventilation and no deaths were reported. The authors concluded that a $6.5 \mathrm{mg} / \mathrm{kg}$ dose of FP produced the optimal balance of efficacy and safety for patients undergoing colonoscopy (Cohen et al 2006; Cohen 2008). While sedation success was high in the FP $8 \mathrm{mg} / \mathrm{kg}$ arm, $25 \%$ of these patients experienced deep sedation during colonoscopy. In contrast, a low 3.8\% incidence of deep sedation was experienced by subjects in both the 5 and $6.5 \mathrm{mg} / \mathrm{kg}$ groups. This highlights the curvilinear doseresponse curve seen with FP. At doses beyond $6.5 \mathrm{mg} / \mathrm{kg}$, small dose escalations produce an exponentially greater risk of deep sedation, thereby making the $8 \mathrm{mg} / \mathrm{kg}$ dose a poor choice for targeting moderate sedation.

Some data on fospropofol are currently available only in abstract and poster form after formal presentation at Digestive Disease Week 2007. Preliminary data from a larger double-blind, multi-center trial of 314 patients undergoing colonoscopy age $\geq 18$ years with ASA risk classes of 1 to 4 demonstrated similar results (Cohen et al 2007a, b; Rex et al 2007b). Patients received either FP $2.0 \mathrm{mg} / \mathrm{kg}$, FP $6.5 \mathrm{mg} / \mathrm{kg}$, or midazolam $0.02 \mathrm{mg} / \mathrm{kg}$ after pretreatment with $50 \mu \mathrm{g}$ of fentanyl citrate. Midazolam was integrated into this study purely as a reference for safety analyses and the study was not powered to show differences between FP and midazolam. Sedation success was defined as a MOAA/S score of $\leq 4$ after administration of sedation and completion of the colonoscopy without use of any alternative sedatives 
or mechanical/manual ventilation. Sedation success was attained in $87 \%, 26 \%$, and $69 \%$ for FP $6.5 \mathrm{mg} / \mathrm{kg}$, FP 2.0 $\mathrm{mg} / \mathrm{kg}$, and $0.02 \mathrm{mg} / \mathrm{kg}$ midazolam group, respectively (Rex et al 2007d). No FP-related serious adverse events or deaths were reported and no patient required either manual or mechanical ventilation. The investigators concluded that the dose of $6.5 \mathrm{mg} / \mathrm{kg}$ is a safe and effective regimen in providing minimal to moderate sedation for patients undergoing colonoscopy.

To measure patient satisfaction, the investigators had patients complete a 6-question satisfaction survey following discharge to gauge satisfaction and comfort on a 10 point scale, 1 indicating low satisfaction or comfort, and 10 indicating high satisfaction or comfort (Cohen et al $2007 b, d)$. Mean satisfaction scores were 9.4 in the FP 6.5 $\mathrm{mg} / \mathrm{kg}$ group and 9.1 in the FP $2 \mathrm{mg} / \mathrm{kg}$ group (Cohen et al 2007d). Mean comfort scores were 9.1 in the FP $6.5 \mathrm{mg} / \mathrm{kg}$ group and 8.7 in the FP $2 \mathrm{mg} / \mathrm{kg}$ group. Physicians also ranked their satisfaction on a similar 10 point scale. Of note, physicians in this study were responsible for the dosing of sedation and remained unblinded to the medications being administered. Physician assessments were taken both at the end of the sedation initiation phase and after completion of the colonoscopy. Mean physician satisfaction scores with the FP $2.0 \mathrm{mg} / \mathrm{kg}$ dosing regimen were only 3.3 after sedation initiation and 4.5 after completion of the procedure. The requirement for alternative rescue sedation in $72 \%$ of patients in the FP $2.0 \mathrm{mg} / \mathrm{kg}$ group was one important factor playing a role in these lower physician satisfaction scores. In contrast, the higher dose of FP $6.5 \mathrm{mg} / \mathrm{kg}$ required alternative sedation in $12 \%$ of patients. This regimen was associated with higher physician satisfaction scores of 7.1 after sedation initiation and 7.7 upon colonoscopy completion. The authors concluded that FP at a dose of $6.5 \mathrm{mg} / \mathrm{kg}$ achieves a high level of patient and physician satisfaction, amnesia during the procedure in most patients, and a willingness of $96 \%$ of patients to be sedated again with FP. This dose was well tolerated, led to clear-headed recovery, with generally mild to moderate paresthesias being the most common observed side effect.

Data on the use of FP for other endoscopic procedures may be applicable to its use for colonoscopy. Vincent and colleagues randomized 252 patients undergoing flexible bronchoscopy to either FP $2.0 \mathrm{mg} / \mathrm{kg}$ or FP $6.5 \mathrm{mg} / \mathrm{kg}$ and both groups received pretreatment with $50 \mu \mathrm{g}$ of fentanyl citrate (Vincent et al 2007). The primary endpoint was sedation success, defined as 3 consecutive MOAA/S scores of $\leq 4$ after administration of sedation, completion of the bronchoscopy without use of any alternative sedatives, and without need for mechanical or manual ventilation. $88.7 \%$ of the FP $6.5 \mathrm{mg} / \mathrm{kg}$ group achieved sedation success compared to only $27.5 \%$ in the $2.0 \mathrm{mg} / \mathrm{kg}$ group. Sedation-related adverse events occurred in $20.1 \%$ of the standard dose FP and $12.6 \%$ of the low dose FP arm. Although no deaths occurred during the study, one patient with a history of severe hypoxemia, COPD, and recurrent pneumonia who was randomized to the FP $6.5 \mathrm{mg} / \mathrm{kg}$ required bag-valve mask ventilation following sedation. As with other trials, the most common adverse reactions were mild, self-limited perineal paresthesias (47.6\%), pruritus (14.7\%), and transient hypoxemia (9.9\%). Paresthesias and pruritus occurred in a similar percentage of patients in each treatment arm. Vincent and colleagues concluded that FP $6.5 \mathrm{mg} / \mathrm{kg}$ is safe and effective in providing titratable sedation for flexible bronchoscopy in a patient population with significant comorbid illness.

\section{Training}

There remains a need for comprehensive sedation training programs to ensure the safety and efficacy of all agents for sedation including non-anesthetist administered propofol and FP (Rex 2006). The American Society for Gastrointestinal Endoscopy has made clear recommendations regarding training requirements to ensure safe administration of deep sedation (American Society for Gastrointestinal Endoscopy Training Committee 2004). Specific requirements include training in advanced cardiac life support, knowledge of the pharmacodynamic and pharmacokinetic principles of each agent used for endoscopic sedation, and education on the indications and contraindications of each agent to be used. Of central importance, practitioners should have a clear understanding of strategies for airway assessment and management including measures to support patients undergoing episodes of airway obstruction or apnea during sedation (Rex 2006). These broad requirements for administration of deep sedation will clearly apply to gastroenterologists administering FP as a means to increase safety of this unique sedative/hypnotic.

\section{Conclusions}

At this time, benzodiazepines remain the most widely utilized class of medication for sedation during endoscopic procedures. While benzodiazepines are effective, they have several specific drawbacks. These include a notable potential for drug-drug interactions, significant metabolic variability between patients, lengthy post-procedural recovery periods, 
and a propensity for producing deeper levels of sedation than intended (Rex 2006; VanNatta and Rex 2006). One recent study found that $45 \%$ of patients undergoing colonoscopy with a combination of a benzodiazepine and opiate for sedation experienced transient deep sedation at some time throughout the procedure (VanNatta and Rex 2006). In light of these notable drawbacks, propofol is often the sedative of choice for gastroenterologists. However, gastroenterologist use of propofol remains quite limited due to the package insert approved by the FDA which specifies that use of propofol should be limited to individuals "trained in the administration of general anesthesia" (AstraZeneca Pharmaceuticals 2004).

FP may be an attractive agent for gastroenterologists interested in realizing the benefits of propofol without the requirement of an anesthesia specialist in the endoscopy suite. Preliminary studies demonstrate that FP $6.5 \mathrm{mg} / \mathrm{kg}$ after intravenous bolus dosing of fentanyl citrate $50 \mu \mathrm{g}$ is well tolerated with a slower pharmacodynamic and pharmacokinetic profile than propofol lipid emulsion. At this dose, FP offers an excellent balance of safety and efficacy while producing high rates of patient and physician satisfaction. The clinical use of FP will necessitate full disclosure to patients of potential side effects of the agent, which include mild to moderate perineal paresthesias and pruritus. Perineal tingling, itching, or burning may occur in up to $85 \%$ of patients and ultimately may limit patient acceptance of this agent for sedation (Pruitt et al 2005). As with all agents employed for endoscopic sedation, adequate monitoring of a patient's cardiovascular and respiratory status remains essential for safe use of FP.

There are several areas that are ripe for further research into the use of FP for sedation during gastroenterologic procedures. To date, there is no published literature on its use during advanced procedures such as endoscopic ultrasound or endoscopic retrograde cholangiopancreatography. These procedures typically require more time to complete and FP may have a different safety and efficacy profile in such settings. Additionally, no large scale trials exist that directly compare outcomes of propofol emulsion with FP for any endoscopic procedure. These data will be essential prior to drawing final conclusions on the relative efficacy of these agents. While FP does appear to have promising applications in sedation during gastroenterologic procedures, we must eagerly await final conclusions from further phase III clinical trials before rendering definitive conclusions as to where FP sits in the armamentarium of endoscopic sedation.

\section{Disclosures}

None of the authors has any conflicts of interest to disclose.

\section{References}

American Society for Gastrointestinal Endoscopy Training Committee. 2004. Training guideline for use of propofol in gastrointestinal endoscopy. Gastrointest Endosc, 60:167-72.

AstraZeneca Pharmaceuticals. 2004. Diprivan ${ }^{\circledR}$ Full Prescribing Information. AstraZeneca Pharmaceuticals, LP.

Cohen LB. 2008. Clinical trial: a dose-response study of fospropofol disodium for moderate sedation during colonoscopy. Aliment Pharmacol Ther. Epub ahead of print. URL: http://www.blackwell-synergy.com/ doi/pdf/10.1111/j.1365-2036.2008.03598.x.

Cohen LB, Rex DK, Kline J, et al. 2007a. Patient and physician satisfaction with fospropofol disodium: results of a phase 3 , randomized, double-blind trial evaluating fospropofol disodium for moderate sedation in patients undergoing colonoscopy. Gastrointest Endosc, 65:AB367.

Cohen LB, Rex DK, Kline J, et al. 2007b. Patient and physician satisfaction with fospropofol disodium: results of a phase 3, randomized, doubleblind trial evaluating fospropofol disodium for moderate sedation in patients undergoing colonoscopy. Digestive Disease Week. Washington D.C., USA. May 19-24, 2007.

Cohen LB, Wang C, Jones JB. 2006. AQUAVAN is safe and effective for minimal to moderate sedation during colonoscopy. Anesthesiology, 105:A1367.

Cohen LB, Hightower CD, Wood DA, et al. 2004. Moderate level sedation during endoscopy: a prospective study using low-dose propofol, meperidine/fentanyl, and midazolam. Gastrointest Endosc, 59:795-803.

Fechner J, Ihmsen H, Hatterscheid D, et al. 2004. Comparative pharmacokinetics and pharmacodynamics of the new propofol prodrug GPI 15715 and propofol emulsion. Anesthesiology, 101:626-39.

Fechner J, Ihmsen H, Hatterscheid D, et al. 2003. Pharmacokinetics and clinical pharmacodynamics of the new propofol prodrug GPI 15715 in volunteers. Anesthesiology, 99:303-13.

Fechner J, Ihmsen H, Schiessl C, et al. 2005. Sedation with GPI 15715, a water-soluble prodrug of propofol, using target-controlled infusion in volunteers. Anesthes Analg, 100:701-6.

Gan TJ. 2006. Pharmacokinetic and pharmacodynamic characteristics of medications used for moderate sedation. Clin Pharmacokinet, 45:855-69.

Gibiansky E, Struys MM, Gibiansky L, et al. 2005. AQUAVAN injection, a water-soluble prodrug of propofol, as a bolus injection: a phase I doseescalation comparison with DIPRIVAN (part 1): pharmacokinetics. Anesthesiology, 103:718-29.

Levitt DG, Schnider TW. 2005. Human physiologically based pharmacokinetic model for propofol. BMC Anesthesiol, 5:4.

Luer MS. 1998. Fosphenytoin. Neurol Res, 20:178-82.

Perron G, Dolbec P, Germain J, et al. 2003. Perineal pruritus after i.v. dexamethasone administration. Can J Anaesth, 50:749-50.

Pruitt RE, Cohen LB, Gibiansky, E. 2005. A randomized open-label, multicenter, dose-ranging study of sedation with Aquavan injection (GPI 15715) during colonoscopy. Gastrointest Endosc, 61:AB111.

Rex DK. 2006. Review article: moderate sedation for endoscopy: sedation regimens for non-anaesthesiologists. Aliment Pharmacol Ther, 24:163-71.

Rex DK, Cohen LB, Kline JK, et al. 2007a. Fospropofol disodium for minimal-to-moderate sedation during colonoscopy produces clearheaded recovery: results of a phase 3, randomized, double-blind trial. Gastrointest Endosc, 65:AB367.

Rex DK, Cohen LB, Kline JK, et al. 2007b. Fospropofol disodium is effective and safe for sedation in patients undergoing colonoscopy: results of a phase 3, randomized double-blind trial. Gastrointest Endosc, 65: AB369. 
Rex DK, Cohen LB, Kline JK, et al. 2007c. fospropofol disodium for sedation during colonoscopy produces clear-headed recovery: results of a phase 3, randomized, double-blind trial. Digestive Disease Week. Washington, D.C., USA. May 19-24, 2007.

Rex DK, Cohen LB, Kline JK, et al. 2007d. Fospropofol disodium is effective and safe for sedation in patients undergoing colonoscopy: results of a phase 3, randomized double-blind trial. Digestive Disease Week. Washington, D.C., USA. May 19-24, 2007.

Rex DK, Heuss LT, Walker JA, et al. 2005. Trained registered nurses/ endoscopy teams can administer propofol safely for endoscopy. Gastroenterology, 129:1384-91.

Schywalsky M, Ihmsen H, Tzabazis A, et al. 2003. Pharmacokinetics and pharmacodynamics of the new propofol prodrug GPI 15715 in rats. Eur J Anaesthesiol, 20:182-90.

Shah A, Fechner J, Struys M, et al. 2007. Differential PK/PD of propofol after intravenous fospropofol and diprivan in healthy subjects. Anesthesiology, 107:A46.
Sipe BW, Rex DK, Latinovich D, et al. 2002. Propofol versus midazolam/ meperidine for outpatient colonoscopy: administration by nurses supervised by endoscopists. Gastrointest Endosc, 55:815-25.

Struys MM, Vanluchene AL, Gibiansky E, et al. 2005. AQUAVAN injection, a water-soluble prodrug of propofol, as a bolus injection: a phase I dose-escalation comparison with DIPRIVAN (part 2): pharmacodynamics and safety. Anesthesiology, 103:730-43.

VanNatta ME, Rex DK. 2006. Propofol alone titrated to deep sedation versus propofol in combination with opioids and/or benzodiazepines and titrated to moderate sedation for colonoscopy. Am J Gastroenterol, 101:2209-17.

Vincent BD, Downie G, Hansbrough J, et al. 2007. Fospropofol disodium is effective and safe for sedation in patients undergoing flexible bronchoscopy: Results of a phase 3, randomized, double-blind, dose-controlled trial. Chest, 132:438a. 